\title{
Peculiar Velocities of Nearby Galaxies
}

\author{
D. Makarov $^{1} \dagger$, I. Karachentsev ${ }^{1}$, B. Tully ${ }^{2}$, L. Makarova ${ }^{1}$, \\ A. Dolphin ${ }^{3}$, S. Sakai ${ }^{4}$, E. Shaya ${ }^{5}$, L. Rizzi ${ }^{2}$, M. Sharina ${ }^{1}$, \\ and V. Karachentseva ${ }^{6}$ \\ ${ }^{1}$ Special Astrophysical Observatory, Russia, ${ }^{2}$ IfA, Univ. of Hawaii, USA, ${ }^{3}$ Steward \\ Observatory, US, ${ }^{4}$ Univ. Of California, Los Angeles, US, ${ }^{5}$ Univ of Maryland, US, \\ ${ }^{6}$ Astronomical Observatory of Kiev, Ukraine
}

Over the last five years, enormous progress in accurate distance measurements for nearby galaxies has been attained. The Hubble Space Telescope allows us to estimate distances of about $7-10 \%$ accuracy using red giant branch (RGB) up to $8 \mathrm{Mpc}$. Now we have very accurate distances for more than 200 galaxies within $\sim 6 \mathrm{Mpc}$.

The Local Volume $(10 \mathrm{Mpc})$ is deep inside the cosmic cell of matter uniformity, the galaxies in the Local region are highly clumped. However, the Hubble flow start just outside the bound of Local Group $\sim 1 \mathrm{Mpc}$ and their random motions are surprisingly small.

We used simple spherically symmetric model to estimate influence of nearby groups on kinematics of Local Volume. This method allows us to measure the masses by deviation of velocities from uniform Hubble flow on scale about $1 \mathrm{Mpc}$ and it is independent from mass estimations by virial theorem. A sample of 229 galaxies with photometric distances less than $7 \mathrm{Mpc}$ have been considered. The turn-around radius for 5 nearby groups have been measured. Derived zero velocity radii are $0.86 \mathrm{Mpc}$ (Local Group), 1.26 Mpc (Cen A), $0.65 \mathrm{Mpc}$ (M 83), 1.15 Mpc (M 81) and 0.78 Mpc (IC 342) which correspond to masses $1.2 \times 10^{12} \mathfrak{M}_{\odot}, 3.7 \times 10^{12} \mathfrak{M}_{\odot}, 5.1 \times 10^{11} \mathfrak{M}_{\odot}, 2.8 \times 10^{12} \mathfrak{M}_{\odot}, 8.7 \times 10^{11} \mathfrak{M}_{\odot}$ respectively. The residual peculiar velocity field has been estimated using the tensor of the local Hubble constant with principal values of 72:76:92. The major axis forms angle about $30^{\circ}$ with direction toward the center of Virgo Cluster.

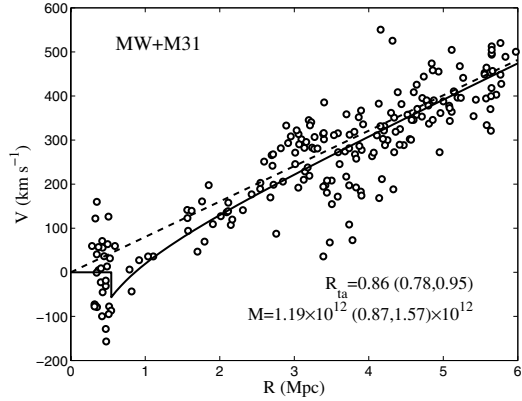

The influence of Local Group on motion of nearby galaxies

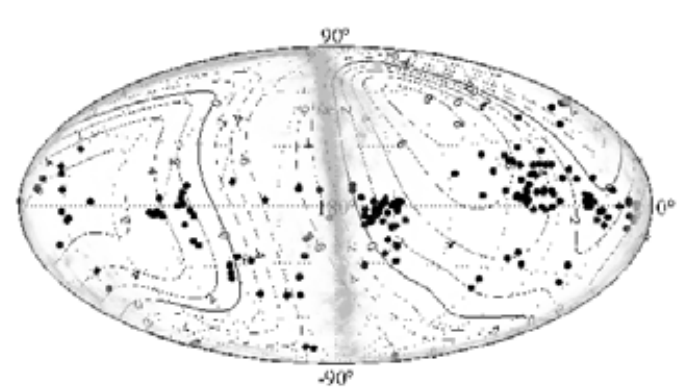

The deviation of Local Volume expantion from the uniform Hubble flow in supergalactic coordinates 